\title{
Torsades de pointes inducidas por sevoflurano
}

\section{Sr. Director:}

La torsade de pointes (TP) es una taquicardia ventricular rápida (160-250 lat/min), caracterizada por complejos QRS de amplitud variable que giran en torno al eje isoeléctrico en forma sinusoidal.

Se presenta el caso de un varón de 39 años, con antecedentes de fumador, intervenido por genu valgo, prótesis de caderas y hemicolectomía izquierda por adenocarcinoma. Fue intervenido de urgencia por una obstrucción intestinal. El quinto día fue trasladado a nuestra unidad de cuidados intensivos (UCI) con insuficiencia respiratoria grave e inestabilidad hemodinámica por peritonitis. Precisó ventilación mecánica, inotrópicos (dobutamina y noradrenalina), sedoanalgesia (propofol y fentanilo) y antibióticos (piperacilina-tazobactam y gentamicina).

El cuarto día de estancia en UCI fue reintervenido por absceso de herida quirúrgica, y se utilizó sevofluorano, fentanilo y dobesilato de cisatracurio. Cinco horas después presentó una TP autolimitada, por lo que se le administró gluconato cálcico, cloruro de potasio y sulfato magnésico. En el electrocardiograma se observó un QTc alargado (737 ms), onda T invertida y morfología de BRD que previamente no existían (fig. 1A). En la analítica se apreciaba hipocalcemia (calcio total, $6,4 \mathrm{mg} / \mathrm{dl}$, e iónico, 0,97 mmol/.), hipopotasemia $(3,3 \mathrm{mmol} / \mathrm{l})$ y magnesemia normal $(2,4 \mathrm{mg} / \mathrm{dl})$. En las $12 \mathrm{~h}$ siguientes persistió el QT largo sin nuevos episodios de TP, pero coincidiendo con la retirada del propofol, se desencadenó una nueva TP (fig. 1B) que cedió con sulfato de magnesio y un bolo de propofol, y se reinició su infusión.

En las siguientes $24 \mathrm{~h}$ presentó cuatro episodios de TP que precisaron descargas eléctricas, ante lo que se decidió colocar marcapasos endocavitario temporal a una frecuencia de 90 lat/min, sin nuevos episodios de TP. Un ecocardiograma descartó lesiones estructurales. Se retiró el marcapasos a las 72 h, con lo que el paciente quedó en ritmo sinusal con intervalo QT normal (425 ms) (fig. 1C). Fue dado de alta de la unidad a los 16 días. En la historia clínica sus electrocardiogramas presentaban un QTc normal.

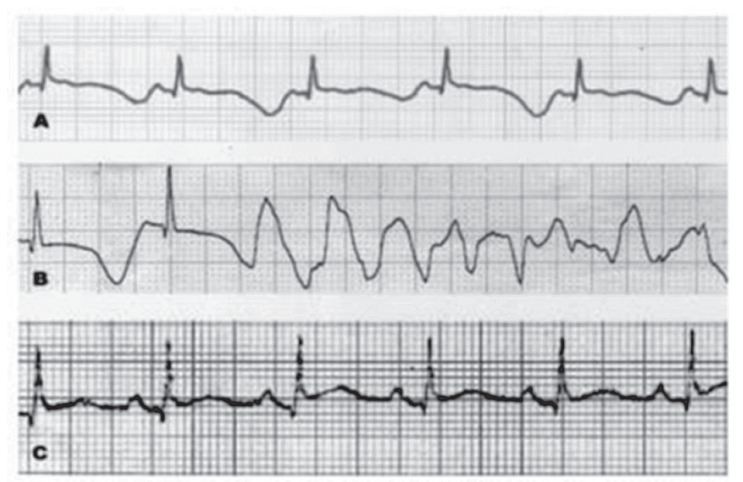

Figura 1. A: QTc alargado. B: comienzo de una de las torsades de pointes. C: QTC normalizado al alta. 
El síndrome de QT largo (SQTL) se caracteriza por un alargamiento del intervalo QT corregido (QTc). El SQTL puede ser congénito o adquirido. La forma adquirida puede deberse a numerosas sustancias, como el sulfato de quinina ${ }^{1}$, el haloperidol ${ }^{2}$ y otros fármacos. Se han descrito factores de riesgo que favorecen las TP inducidas por drogas: sexo femenino, hipopotasemia, bradicardia, hipomagnesemia, fallo cardíaco congestivo, terapia con digital, etc. ${ }^{3}$.

El aumento del intervalo QTc es resultado de una alteración en la repolarización ventricular, y puede desencadenar una TP. Su tratamiento consiste en la suspensión del agente causal y la modificación de los factores de riesgo. Además, debemos administrar sulfato de magnesio y acelerar la frecuencia cardíaca con isoproterenol o un marcapasos.

El sevoflurano interfiere en varios canales iónicos cardíacos y puede causar alteraciones en la repolarización ventricular. Se ha observado que prolonga el QTc en presencia de agentes que alarguen el $\mathrm{QT}^{4}, \mathrm{y}$ en los que no presentan factores de riesgo ${ }^{5}$.

El propofol se considera adecuado para pacientes con SQTL que precisen sedación. Se ha descrito la resolución de taquicardias ventriculares no sostenidas con bolos de propofol, que incluso permanecen suprimidas en los pacientes que precisan infusión continua, lo que se explica gracias a un bloqueo simpático ${ }^{6}$. En nuestro caso la primera de las TP cedió tras un bolo de propofol, aunque posteriormente, pese a encontrarse con infusión continua, sufrió varios episodios más.

En el caso presentado no había un SQTL congénito y se descartó afección estructural cardíaca. Hay una relación temporal entre la administración de sevoflurano y la prolongación del QTc, en que la hipopotasemia pudo ejercer como factor predisponente.

JUAN CARLOS RODRÍGUEZ-BORREGAN, FRANCISCO JAVIER BURON-MEDIAVILLA, VIRGINIA ARNAIZARNAIZ Y JOSÉ MARÍA MARCO-MORENO

Servicio de Medicina Intensiva. Hospital Universitario Marqués de Valdecilla. Santander. Cantabria. España.

\section{BIBLIOGRAFÍA}

1. Ramírez A, Galván JM. Taquicardia ventricular tipo torsades de pointes en un paciente con intoxicación digitálica en tratamiento crónico con sulfato de quinina. Med Intensiva. 2007;31: 106-7.

2. Herrero-Hernández R, Cidoncha-Gallego M, Herrero-De Lucas E, Jiménez-Lendínez M. Haloperidol por vía intravenosa y torsade de pointes. Med Intensiva. 2004;28:89.

3. Roden DM. Drug-induced prolongation of the QT interval. N Engl J Med. 2004;350:1013-22.

4. Paventi S, Santevecchi A, Ranieri R. Effects of sevoflurane versus propofol on QT interval. Minerva Anestesiol. 2001;67:63740.

5. Kuenszberg E, Loeckinger A, Kleinsasser A, Lindner KH, Puehringer F, Hoermann C. Sevoflurane progressively prolongs the QT interval in unpremedicated female adults. Eur J Anaesthesiol. 2000;17:662-4.

6. Burjorjee JE, Milne B. Propofol for electrical storm; a case report of cardioversion and suppression of ventricular tachycardia by propofol. Can J Anaesth. 2002;49:973-7. 